\title{
Sinapic Acid Ameliorates Oxidative Stress, Inflammation, and Apoptosis in Acute Doxorubicin-Induced Cardiotoxicity via the NF- $\kappa$ B-Mediated Pathway
}

\author{
Yousef A. Bin Jardan, ${ }^{1}$ Mushtaq Ahmad Ansari $\left(\mathbb{D},{ }^{2}\right.$ Mohammad Raish $\left(\mathbb{D},{ }^{1}\right.$ \\ Khalid M. Alkharfy, ${ }^{3}$ Abdul Ahad $\odot{ }^{1},{ }^{1}$ Fahad I. Al-Jenoobi $\odot{ }^{1},{ }^{1}$ Nazrul Haq, \\ Mohd Rashid Khan, ${ }^{2}$ and Ajaz Ahmad $\mathbb{D}^{3}$ \\ ${ }^{1}$ Department of Pharmaceutics, College of Pharmacy, King Saud University, Riyadh 11451, Saudi Arabia \\ ${ }^{2}$ Department Pharmacology and Toxicology, College of Pharmacy, King Saud University, Riyadh 11451, Saudi Arabia \\ ${ }^{3}$ Department Clinical Pharmacy, College of Pharmacy, King Saud University, Riyadh 11451, Saudi Arabia \\ Correspondence should be addressed to Mushtaq Ahmad Ansari; muansari@ksu.edu.sa and Ajaz Ahmad; aajaz@ksu.edu.sa
}

Received 8 October 2019; Revised 4 February 2020; Accepted 19 February 2020; Published 10 March 2020

Academic Editor: Klaus Wimmers

Copyright (C) 2020 Yousef A. Bin Jardan et al. This is an open access article distributed under the Creative Commons Attribution License, which permits unrestricted use, distribution, and reproduction in any medium, provided the original work is properly cited.

\begin{abstract}
In the present study, we explored SA's activity against DOX-induced cardiotoxicity and revealed its underlying mechanisms. Male Wistar rats (weight, 190-210g; $n=6$ ) were randomly divided into four groups: group I, normal control; group II, DOX $15 \mathrm{mg} / \mathrm{kg}$ via intraperitoneal (ip) route; group III, administered DOX+SA $20 \mathrm{mg} / \mathrm{kg}$; and group IV, administered DOX+captopril (CAP $30 \mathrm{mg} / \mathrm{kg})$. SA and CAP were administered orally for seven days, and DOX $(15 \mathrm{mg} / \mathrm{kg})$ was injected intraperitoneally an hour before SA treatment on the fifth day. Forty-eight hours after DOX administration, animals were anesthetized and sacrificed for molecular and histology experiments. SA significantly mitigated the myocardial effects of DOX, and following daily administration, it reduced serum levels of lactate dehydrogenase (LDH) and creatine kinase isoenzyme-MB to near normal values. Levels of oxidative stress markers, glutathione-peroxidase, superoxide dismutase, and catalase, in the cardiac tissue were significantly increased, whereas malondialdehyde levels decreased after SA treatment in DOX-administered rats. Furthermore, DOX caused an inflammatory reaction by elevating the levels of proinflammatory cytokines, tumor necrosis factor- $\alpha$ (TNF- $\alpha$ ), interleukin-1 $\beta$ (IL-1 $\beta$ ), and endothelin- (ET-) 1 , as well as nuclear factor kappa-B (NF- $\kappa$ B) expression. Daily administration of SA significantly repressed TNF- $\alpha$, IL- $1 \beta$, ET-1, and NF- $\kappa$ B levels. caspase- 3 and Bax expression, bcl-2-like protein and caspase- 3 activities and levels. Overall, we found that SA could inhibit DOX-induced cardiotoxicity by inhibiting oxidative stress, inflammation, and apoptotic damage.
\end{abstract}

\section{Introduction}

Since 1960, doxorubicin (DOX, Adriamycin) has been a potent chemotherapeutic agent. DOX belongs to the class of anthracyclines and is derived from Streptomyces peucetius $[1,2]$. This potent anticancer agent is used as a chemotherapy for various malignancies such as lymphomas; leukemia; Kaposi's sarcoma; and breast, gastric, and esophageal carcinomas. However, owing to the lethal cardiotoxicity of DOX, its use is restricted [3]. DOX-induced cardiotoxicity is associated with oxidative stress, apoptosis, and inflammation $[4,5]$.

Several reports have indicated that DOX induces inflammation in cardiac muscles and vasculature through nuclear factor kappa-B (NF- $\kappa \mathrm{B})$, which is a critical regulator of inflammatory and immunological reactions. DOX is reduced to semiquinone which generates reactive oxygen species (ROS) such as superoxide and hydrogen peroxide [6] and depletes glutathione peroxidase (GPx) and catalase (CAT), thereby reducing the myocardium's ability to eliminate 
ROS. Furthermore, DOX chelates iron, and the subsequent complex catalyzes the transformation of peroxide radicals to reactive hydroxyl radicals, resulting in oxidative and mitochondrial damages to the myocardium $[6,7]$. The potential implication of apoptosis in DOX-induced cardiotoxicity is via the intrinsic apoptotic pathway $[4,8]$. In addition, this agent plays a crucial role in chemotherapy but as previously mentioned, cardiotoxicity limits its use. Natural antioxidants have been adopted as a chemoprotective approach to reverse cardiotoxicity [9]. Sinapic acid (SA) is a potent free radical scavenging agent that can inhibit lipid peroxidation and restore endogenous antioxidants [10-12]. Sinapic acid and its cardioprotective role against ischemia/reperfusion, isoprotenol, and arsenic have also been previously reported $[13,14]$. Hence, we postulated that SA can efficiently repress DOX-induced cardiotoxicity and sought to explore SA's activity against DOX-induced cardiotoxicity and reveal its underlying mechanisms in the present investigation.

\section{Materials and Methods}

2.1. Drugs and Chemicals. SA, DOX, and CAP were procured from Sigma-Aldrich (USA). Antibodies against NF- $\kappa \mathrm{B}$ (p65), caspase-3, Bax, Bcl-2, $\beta$-actin, and HRP secondary antibodies were obtained from Santa Cruz (USA). The NE-PER Extraction Kit was purchased from Pierce Biotechnology (USA). Rat TNF- $\alpha$, IL- $1 \beta$, and myeloperoxidase (MPO) ELISA kits were bought from R\&D Systems (USA).

2.2. Experimental Design. Adult male Wistar rats (weight, 190-210g) were obtained from the animal facility of King Saud University, Riyadh, Saudi Arabia. The experiment proposal was authorized by the Ethics Committee of the Experimental Animal Care Society, King Saud University, Saudi Arabia (KSU-SE-19-40). Animals were arbitrarily and equally divided into four categories ( $n=6$ animals/group): group I, normal control provided with normal saline; group II, administered DOX $15 \mathrm{mg} / \mathrm{kg}$ via intraperitoneal (ip) route; group III, administered DOX+SA $20 \mathrm{mg} / \mathrm{kg}$; and group IV, administered DOX+captopril (CAP; $30 \mathrm{mg} / \mathrm{kg}$ ). CAP was used as the standard drug for cardiac protection as per earlier reports $[15,16]$. SA and CAP were administered orally for seven days, and DOX (15 mg/kg) was injected intraperitoneally an hour before SA treatment on the fifth day. Forty-eight hours after DOX administration, animals were anesthetized with ketamine $100 \mathrm{mg} / \mathrm{kg}$ and $10 \mathrm{mg} / \mathrm{kg}$, ip [17]. Blood samples were collected from all groups, and serum separation was carried out at $5000 \mathrm{rpm}$ for $15 \mathrm{~min}$. All rats were sacrificed, and their hearts were harvested for molecular and histopathological examinations.

2.3. Serum Biochemical Indices. Lactate dehydrogenase $(\mathrm{LDH})$ and creatinine kinase $\mathrm{MB}$ fraction (CK-MB) levels were estimated in the rat serum with commercial calorimetric kits (Merck). Nitric oxide (NO) and endothelin-1 (ET-1) levels were assessed.

2.4. Oxidative and Antioxidant Indices. Cardiac tissues were balanced to make a $10 \%(w / v)$ RIPA-buffered homogenate using T 25 Digital ULTRA-TURRAX ${ }^{\circledR}$. After centrifugation of the homogenate, the upper layer was collected for biochemical analysis. Protein level was quantified by the Lowry method [18]. The level of MDA, in the cardiac tissue, was examined by using a lipid peroxidation (malondialdehyde (MDA)) assay kit (Sigma-Aldrich, St. Louis, MO, USA). Lipid peroxidation was determined by the reaction of MDA with thiobarbituric acid (TBA) to form a colorimetric product, proportional to the MDA present. The intensity of the color was measured spectrophotometrically at $532 \mathrm{~nm}$. Glutathione peroxidase (GPx) activity was estimated by a glutathione peroxidase kit specific for rats, according to the manufacturer's protocol (ZellBio GmbH, Germany). The activity of glutathione peroxidase (GPx) was determined by NADPH-coupled assay according to the method of Lawrence and Burk [19], and this enzyme catalyzes the oxidation of glutathione by hydrogen peroxide. The oxidation of NADPH was followed spectrophotometrically at $340 \mathrm{~nm}$. SOD activity was estimated by Beauchamp and Fridovich [20]. The reaction mixture consisted of $0.5 \mathrm{ml}$ of cardiac PMS, $1 \mathrm{ml}$ of $50 \mathrm{mM}$ sodium carbonate, $0.4 \mathrm{ml}$ of $25 \mu \mathrm{M} \mathrm{NBT}$, and $0.2 \mathrm{ml}$ of $0.1 \mathrm{mM}$ EDTA. The reaction was initiated by an addition of $0.4 \mathrm{ml}$ of $1 \mathrm{mM}$ hydroxylamine-hydrochloride. The change in absorbance was recorded at $560 \mathrm{~nm}$. Determination of catalase (CAT) activity was examined by a Catalase (CAT) assay kit specific for rats, according to the manufacturer's protocol (abcam. ab83464). Catalase (CAT) level was assessed using $\mathrm{H}_{2} \mathrm{O}_{2}$ as the substrate by the methods of Beers and Sizer [21]. The disappearance of $\mathrm{H}_{2} \mathrm{O}_{2}$ was followed spectrophotometrically at $240 \mathrm{~nm}$.

2.5. Cytokine and Inflammatory Marker. TNF- $\alpha$, IL- $1 \beta$, and MPO contents were examined in the cardiac tissue using ELISA kits (R\&D Systems). Absorbance was read at $450 \mathrm{~nm}$.

2.6. Preparation of Nuclear and Total Protein Extracts. Nuclear and cytosol proteins were isolated with the NEPER Kit (Pierce Biotechnology). Western blot was carried out as per protocols of Towbin et al. [22]. In brief, $25 \mu \mathrm{g}$ of protein was transferred to PVDF membranes, blocked in $4 \%$ skimmed milk in TBS (1\% Tween 20), and incubated at $4{ }^{\circ} \mathrm{C}$ overnight with the antibodies, cleaved caspase- $3, \mathrm{Bax}$, $\mathrm{Bcl}-2, \mathrm{NF}-\kappa \mathrm{B}$ (p65), $\mathrm{I} \kappa \mathrm{B} \alpha$, and $\beta$-actin. This was followed by several washings with $1 \%$ Tween and TBS. Incubation was then performed with secondary antibodies for $2 \mathrm{~h}$ at room temperature. Bands were observed using Luminata ${ }^{\mathrm{TM}}$ Western Chemiluminescent HRP Substrates (Millipore, Billerica, MA, USA). A densitometric analysis of the immunoblots (LI-COR C-Di-Git Blot Scanners (Lincoln, NE, USA)) was also performed.

2.7. Histological Analysis. Cardiac tissues were fixed with $10 \%$ formalin, dehydrated in a gradient series of alcohol, and embedded in paraffin blocks. Tissues were then sliced into $4 \mu \mathrm{m}$ sections, stained with hematoxylin and eosin ( $\mathrm{H} \& \mathrm{E})$, and examined under a light microscope.

2.8. Statistical Analysis. All data are expressed as mean \pm SEM. Statistical analysis was performed using the one-way analysis of variance (ANOVA). 
TABLE 1: Effects of SA on body weight and heart body weight ratio.

\begin{tabular}{lcccc}
\hline Groups & Initial body weight & Final body weight & Gain in body weight & Heart body weight ratio \\
\hline Normal control & $213.00 \pm 1.71$ & $225.20 \pm 1.71$ & $12.20 \pm 2.19$ & $4.26 \pm 0.05$ \\
DOX $15 \mathrm{mg} / \mathrm{kg}$ & $221.80 \pm 1.65^{*}$ & $187.40 \pm 0.92^{*}$ & $-34.40 \pm 1.57$ & $3.86 \pm 0.03$ \\
DOX $15 \mathrm{mg} / \mathrm{kg}+$ SA $20 \mathrm{mg} / \mathrm{kg}$ & $224.00 \pm 1.58^{*}$ & $197.20 \pm 1.82^{* \#}$ & $-26.80 \pm 2.76$ & $4.25 \pm 0.08$ \\
DOX $15 \mathrm{mg} / \mathrm{kg}+$ CAP $30 \mathrm{mg} / \mathrm{kg}$ & $224.40 \pm 1.96^{*}$ & $200.20 \pm 1.15^{* \#}$ & $-24.20 \pm 1.88$ & $4.22 \pm 0.05$ \\
\hline
\end{tabular}

* denotes significant differences compared to the control group $(p<0.05)$; ${ }^{*}$ denotes significant differences compared to the DOX group $(p<0.05)$.

TABLE 2: Effects of SA and CAP on serum LDH and CK-MB levels in the different rat groups. Values are expressed as mean \pm SEM.

\begin{tabular}{lcc}
\hline Groups & LDH (U/l) & CK-MB (U/l) \\
\hline Normal control & $199.73 \pm 5.37$ & $125.82 \pm 2.83$ \\
DOX $15 \mathrm{mg} / \mathrm{kg}$ & $627.42 \pm 18.88^{*}$ & $254.36 \pm 4.14^{*}$ \\
DOX $15 \mathrm{mg} / \mathrm{kg}+$ SA $20 \mathrm{mg} / \mathrm{kg}$ & $217.47 \pm 2.84^{* \#}$ & $157.52 \pm 2.98^{* \#}$ \\
DOX $15 \mathrm{mg} / \mathrm{kg}+$ CAP $30 \mathrm{mg} / \mathrm{kg}$ & $211.72 \pm 2.66^{* \#}$ & $152.87 \pm 3.47^{* \#}$ \\
\hline${ }^{*}$ denotes significant differences compared to the control group $(p<0.05) ;$ \\
denotes significant differences compared to the DOX group $(p<0.05)$.
\end{tabular}

\section{Results}

This section may be divided by subheadings. It should provide a concise and precise description of the experimental results, their interpretation, and the experimental conclusions that can be drawn.

3.1. Cardiotoxicity Indices. Although the intake of DOX resulted in a reduction in body weight of treated rats, this alteration was not statistically different from that of normal control rats. Absolute body weight and heart index, however, were significantly reduced by $16.78 \%$ and $9.40 \%$, respectively, in the DOX-administered group compared to the corresponding values in the control group. When DOXadministered animals were pretreated with SA and CAP, absolute weight was, respectively, enhanced by $5.23 \%$ and $6.83 \%$, whereas heart index was increased by $10.37 \%$ and $9.14 \%$ compared to those in the control group (Table 1).

3.2. Cardiotoxicity Biochemical Indices. DOX-administered rats displayed a significant increase in the levels of $\mathrm{LDH}$ (214.13\%) and CK-MB (102.14\%) compared to those in normal rats. In contrast, animals treated with SA and CAP displayed a significant reduction in these parameters ( $\mathrm{LDH}$, $65.33 \%$ and $66.25 \%$; and CK-MB, 38.07\% and 39.90\%) unlike animals in the DOX group ( $p<0.05$; Table 2$)$.

3.3. Effects of SA and CAP on NO and ET-1 Levels. ET-1 levels were significantly increased (59.51\%; $p<0.001$ ), whereas those of NO were significantly reduced (66.76\%) in DOXadministered rats compared to those in normal rats. Treating DOX-induced rats with SA and CAP resulted in a significant reduction in ET-1 levels (29.13\% and $26.81 \%$, respectively) and a significant increase in NO levels $(100.07 \%$ and $119.075 \%$, respectively) compared to those in rats treated with DOX alone (Table 3).
TABLE 3: Effects of SA and CAP on NO and ET-1 levels.

\begin{tabular}{lcc}
\hline Group & ET-1 $(\mathrm{pg} / \mathrm{mg})$ & NO $(\mu \mathrm{M})$ \\
\hline Normal control & $128.37 \pm 1.72$ & $21.072 \pm 1.17$ \\
DOX $15 \mathrm{mg} / \mathrm{kg}$ & $204.77 \pm 1.08^{*}$ & $7.003 \pm 0.44^{*}$ \\
DOX $15 \mathrm{mg} / \mathrm{kg}+$ SA $20 \mathrm{mg} / \mathrm{kg}$ & $145.10 \pm 2.02^{* \#}$ & $13.622 \pm 0.27^{* \#}$ \\
DOX $15 \mathrm{mg} / \mathrm{kg}+\mathrm{CAP} 30 \mathrm{mg} / \mathrm{kg}$ & $149.85 \pm 2.05^{* \#}$ & $14.919 \pm 0.33^{* \#}$ \\
\hline${ }^{*}$ denotes significant differences compared to the control group $(p<0.05) ;{ }^{*}$ \\
denotes significant differences compared to the DOX group $(p<0.05)$.
\end{tabular}

3.4. Effects of SA on the Levels of Lipid Peroxidation and Antioxidant Enzymes. Lipid peroxidation and antioxidant enzyme activities were measured in all groups (Table 4). DOX was found to induce lipid peroxidation as demonstrated by the increase in MDA level (69.57\%) compared to that observed in normal group rats. The SA- and CAPadministered rats demonstrated a significant reduction in MDA levels $(63.20 \%$ and $65.32 \% \mathrm{nmol} / \mathrm{mg}$, respectively) compared to those in DOX-induced rats $(p<0.01$; Table 4$)$. Similarly, the DOX-administered group exhibited a significant decrease in SOD (58.28\%), GSH (60.66\%), and CAT $(66.69 \%)$ levels compared to those in the normal group $(p<0.01)$. Treatment with SA and CAP significantly restored the depletion of antioxidant enzymes such as SOD $(44.35 \%$ and $27.56 \%$; $p<0.01, p<0.01)$, GSH $(43.16 \%$ and $59.64 \%$; $p<0.001, p<0.001)$, and CAT (112.81\% and $112.59 \%$; $p<$ $0.001)$ compared to the levels found in DOX-administered rats (Table 4$)$.

3.5. Effects of $S A$ and CAP on the Levels of TNF- $\alpha, I L-1 \beta$, and $M P O$. The levels of the inflammatory cytokines, TNF- $\alpha$ and IL- $1 \beta$, and inflammatory marker, MPO, were determined in the cardiac tissue of all groups as illustrated in Figure 1. DOX-treated rats showed significant increases in TNF- $\alpha$ (349.61\%), IL-1 $\beta$ (228.007\%), and MPO (53.98\%) levels in this tissue compared to those in normal rats $(p<0.01)$. However, treatment with SA and CAP significantly downregulated the inflammatory responses of TNF- $\alpha$ (36.36\% and $38.41 \%$ ), IL- $\beta$ ( $43.25 \%$ and $40.55 \%)$, and MPO $(19.08 \%$ and $20.62 \%$ ) compared to the levels observed in DOXadministered rats.

3.6. Effects of SA and CAP on the Expression of Bax, Caspase3, Bcl-2, and NF- $\kappa B$. Expression of the apoptotic proteins Bax and caspase-3 significantly increased, whereas that of the antiapoptotic protein $\mathrm{Bcl}-2$ significantly reduced in the DOX group compared to that found in the normal group. Pretreatment with SA and CAP significantly enhanced the 
TABLE 4: Effects of sinapic acid (SA) and captopril (CAP) on the levels of lipid peroxidation and antioxidant enzymes. Values are expressed as mean \pm SEM.

\begin{tabular}{|c|c|c|c|c|}
\hline Group & MDA (nmol/mg) & $\mathrm{SOD}(\mathrm{U} / \mathrm{mg})$ & GSH (U/mg) & CAT (U/mg) \\
\hline Normal control & $38.01 \pm 1.27$ & $35.44 \pm 0.82$ & $4.48 \pm 0.08$ & $8.12 \pm 0.53$ \\
\hline DOX $15 \mathrm{mg} / \mathrm{kg}$ & $124.97 \pm 2.67$ & $14.78 \pm 0.38$ & $1.76 \pm 0.072$ & $2.70 \pm 0.21$ \\
\hline DOX $15 \mathrm{mg} / \mathrm{kg}+\mathrm{SA} 20 \mathrm{mg} / \mathrm{kg}$ & $45.92 \pm 2.21^{*}$ & $21.33 \pm 0.82^{* \#}$ & $2.52 \pm 0.12$ & $5.75 \pm 0.22^{* \#}$ \\
\hline DOX $15 \mathrm{mg} / \mathrm{kg}+\mathrm{CAP} 30 \mathrm{mg} / \mathrm{kg}$ & $43.36 \pm 1.52^{* \#}$ & $18.86 \pm 0.68^{* \#}$ & $2.81 \pm 0.08$ & $5.74 \pm 0.31^{* \#}$ \\
\hline
\end{tabular}

${ }^{*}$ denotes significant differences compared to the control group $(p<0.05) ;{ }^{*}$ denotes significant differences compared to the DOX group ( $\left.p<0.05\right)$.

expression of $\mathrm{Bcl} 2$ and reduced the expression of Bax and caspase- 3 compared to those observed in the DOX group, thereby reducing the apoptosis-induced cardiac injuries (Figures 2(a)-2(c)). In the DOX group, a relatively significant activation of NF- $\kappa \mathrm{B}$ occurred in the cardiac tissue compared to that observed in rats in the normal group. In contrast, pretreating DOX-administered animals with SA and CAP significantly attenuated NF- $\kappa$ B expression (Figure $2(\mathrm{~d})$ ).

3.7. Histopathological Evaluation. Myocardium tissues from normal rats had regular cell distribution and myocardium architecture (Figure 3(a)). Histological examination of the hearts from DOX-administered animals revealed a significant loss of myofibrils and wavy fibers (Figure 3(b)). Pretreatment with $20 \mathrm{mg} / \mathrm{kg}$ of SA and CAP, however, nearly preserved the normal architecture of the myocardium (Figures 3(c) and 3(d)).

\section{Discussion}

DOX is a life-threatening chemotherapeutic agent used for cancer treatment. Because it results in severe cardiotoxicity, its clinical utility is thus limited $[1,23]$. DOX induces cardiomyopathy, and if this advances, a poor prognosis occurs, often leading to fatality [24]. Oxidative stress, inflammation, and apoptosis play a significant role in DOX-induced cardiotoxicity $[4,23,24]$. In addition, DOX-induced cardiomyopathy is the primary cause of heart failure. SA possesses potent antioxidant and anti-inflammatory activities; therefore, it is postulated that SA could also have defensive effects against doxorubicin-induced cardiotoxicity. In the present study, we employed a DOX-induced cardiotoxicity rodent model to evaluate the cardioprotective effect of SA in DOXinduced cardiotoxicity using biochemical, morphometric, and histological parameters. No mortality was found in the groups included in the study; however, a reduction in body weight, heart weight, and heart index occurred in the DOXinduced groups. We, then, examined the probable molecular mechanisms underlying the cardioprotective effects of SA. Free radical-induced oxidative stress has been reported as main contributing factors to the DOX-induced deformation of heart tissues $[25,26]$. DOX anthracycline structure has been indicated to release enzymatic and nonenzymatic redox cycle liberation of ROS from molecular oxygen [27]. Free radical scavenging, therefore, delivers important ways to protect against DOX-induced oxidative injury. Free radical scavenging, therefore, delivers important ways to protect against DOX-induced oxidative injury. Sinapic acid (SA, 3,5-dimethoxy-4-hydroxycinnamic acid) is a phytoconstituent extensively present in spices, berry fruits citrus, vegetables, oilseed crops, and cereals [28] and is known to possess various pharmacological activities, such as antioxidant, antihypertensive, antimicrobial, anti-inflammatory, antianxiety, and anticancer activities. SA is richly present in plants of the Brassicaceae family [29]. It has potent ROS/RNS- and free radical-scavenging activity to protect against tissue damage $[30,31]$. Being its potent antioxidant and anti-inflammatory activities, we hypothesized that SA could also have defensive effects against doxorubicin-induced cardiotoxicity.

Pretreatment with SA and CAP significantly ameliorated the loss in these parameters, reflecting the cardioprotection that they can elicit and align with the results of previous studies [32-34]. CAP is used as a standard cardioprotective agent against DOX-induced cardiotoxicity [15]. The prevailing experimental indication suggests that DOX influences the accumulation of free radicals in cardiac tissue, causing injuries to intracellular components as well as the myocardium and its membranes [2]. Injuries to the myocardial membranes result in the release of $\mathrm{LDH}$ and $\mathrm{CK}-\mathrm{MB}$ in serum or plasma. $\mathrm{LDH}$ and $\mathrm{CK}-\mathrm{MB}$ remain the standard biomarkers for cardiac injuries. Increases in $\mathrm{LDH}$ and serum CK-MB levels suggest that DOX causes cardiac injuries in cellular membranes, which result from pretreatment with SA and CAP. This elevation in LDH and CK-MB aligns with that found in previous investigations [5, 23]. The antiischemic effect of SA and CAP on cardiac tissues was determined by TTC staining [35]. The DOX-administered groups exhibited a significant increase in \% area of necrosis, and this was significant in animals pretreated with SA and CAP.

Present literature suggests that DOX-induced oxidative stress is due to accumulation of free radicals in cardiac tissues. Cardiac tissues are particularly vulnerable to free radicle injuries as they contain low levels of antioxidant enzymes such as SOD, GSH, and CAT. Further, DOX displays high affinity toward phospholipid components of mitochondrial membranes in cardiac cells, leading to its accumulation in heart tissues $[1,6,36]$. DOX-induced mitochondrial injuries are critical as they presumably exhibit life-threatening adverse effects on the heart's contractile function. Therefore, we employed a protocol that would initiate DOX-induced oxidative stress prior to SA and CAP intervention to explore the extent of progressive cardiac damage. CAP has been used as reference standard. Captopril is a potent, competitive inhibitor of angiotensin-converting enzyme (ACE), the enzyme responsible for the conversion of angiotensin I (ATI) to angiotensin II (ATII). ATII regulates blood pressure and is a key 


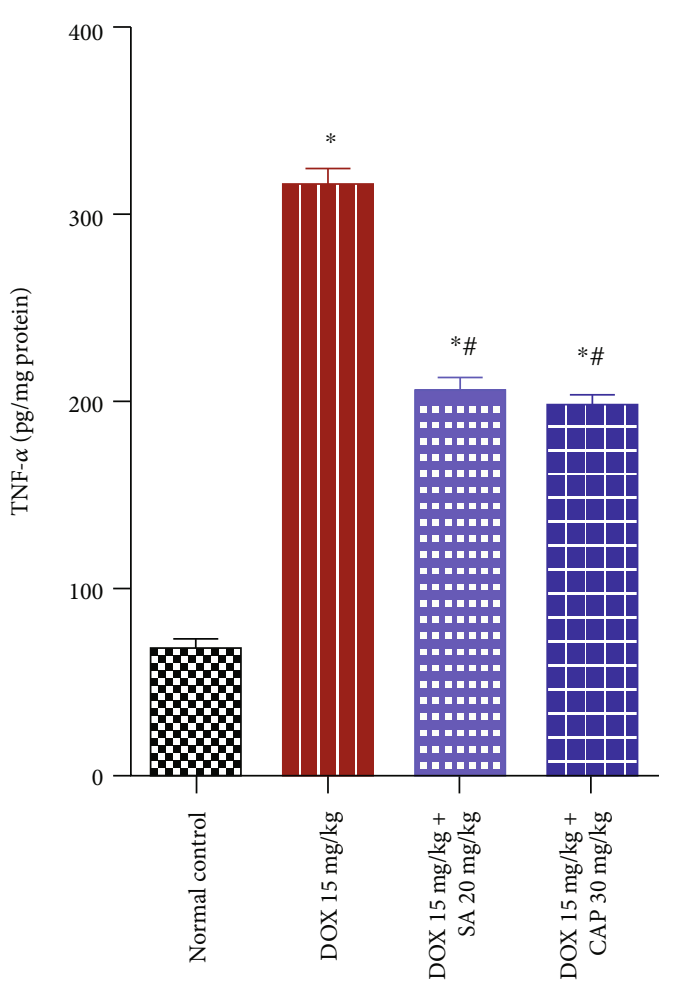

(a)

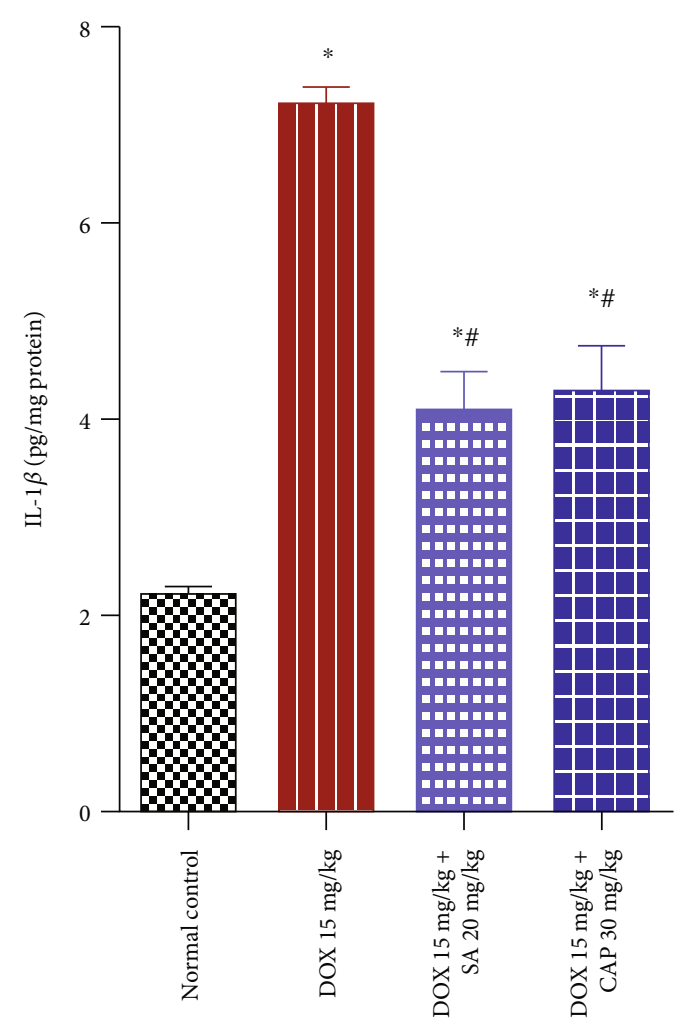

(b)

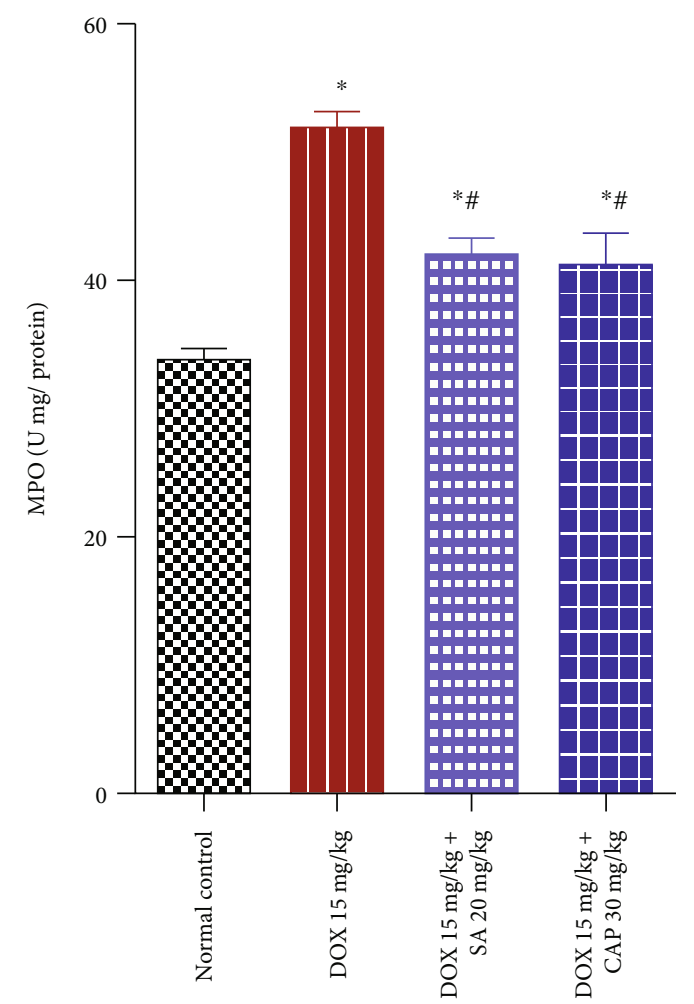

(c)

Figure 1: Effect of sinapic acid (SA) on the levels of proinflammatory cytokines (TNF- $\alpha$, IL-1 $\beta$, and MPO) in control and experimental rats. The results are presented as mean \pm SEM with six animals per group. ${ }^{*}$ denotes significant differences compared to the control group $(p<0.05)$; ${ }^{\#}$ denotes significant differences compared to the DOX group $(p<0.05)$. 


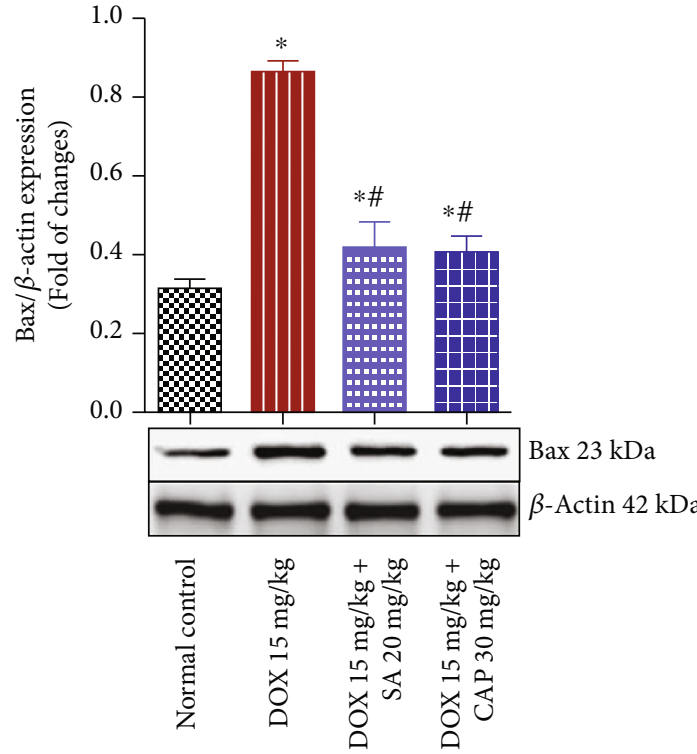

(a)

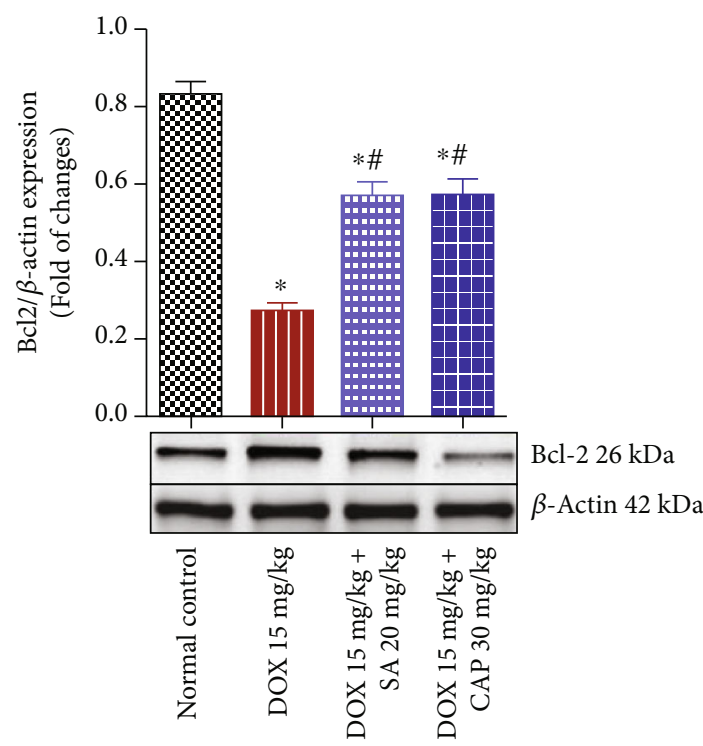

(c)

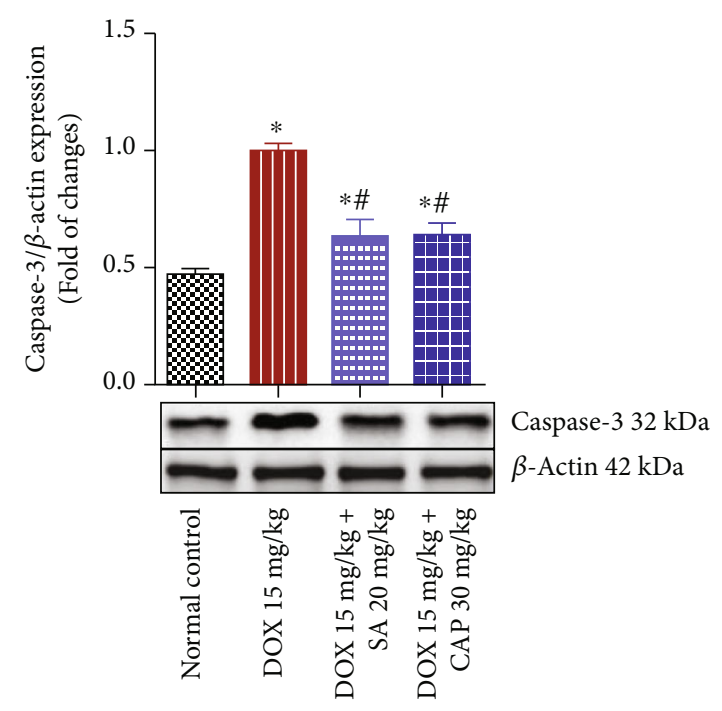

(b)

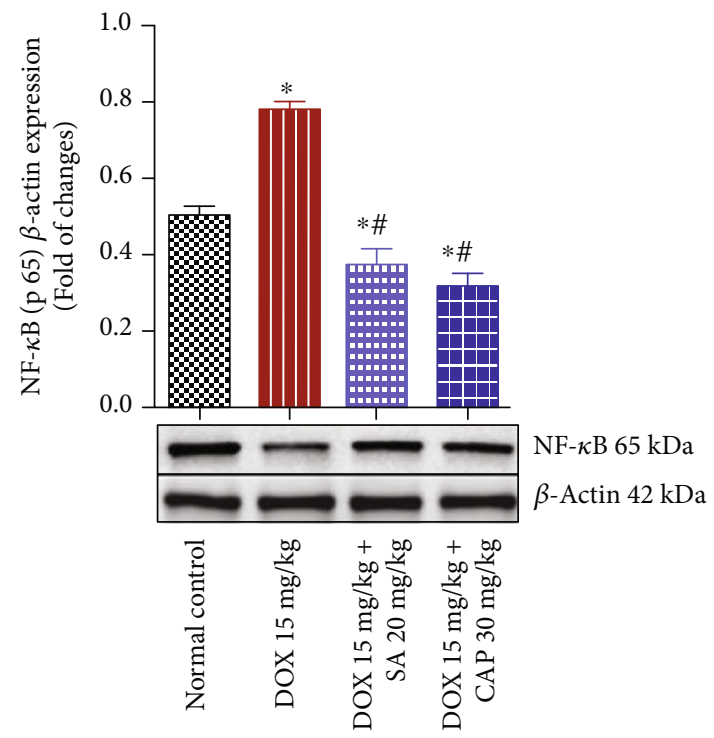

(d)

Figure 2: Effect of sinapic acid (SA) on (a) Bax, (b) caspase-3, (c) Bcl-2, and (d) NF- $\kappa$ B protein expression in control and experimental rats. The results are presented as mean \pm SEM with six animals per group. ${ }^{*}$ denotes significant differences compared to the control group $(p<0.05)$; \# denotes significant differences compared to the DOX group $(p<0.05)$.

component of the renin-angiotensin-aldosterone system (RAAS). Captopril is used to treat high blood pressure (hypertension), congestive heart failure, kidney problems caused by diabetes, and to improve survival after a heart attack $[16,37]$.

Pretreatment with SA and CAP could minimize DOXinduced cardiotoxicity indices via several routes. Lipid peroxidation (MDA) increased in the cardiac membranes of DOX-administered animals. MDAs are the end products of lipid peroxidation; thiobarbituric acid (TBA) is reacted with MDA, which is resulting in a color compound, which can be determined spectrophotometrically. An elevation in MDA level suggests an enhancement in oxidative stress and a reduction in antioxidant enzymes $[6,38]$. In the current investigation, there was a marked elevation in lipid peroxidation and MDA levels and depletion in the antioxidant defenses (SOD, GSH, and CAT) following DOX-induced cardiotoxicity. SA has been reported to exert detrimental effects on lipid peroxidation and oxidative stress, but it is also capable of controlling the elevation in MDA and depletion of antioxidant defenses to moderate extents $[12,39]$. SA and CAP pretreatment significantly reduced lipid peroxidation (MDA) and restored the depleted antioxidant enzymes (SOD, GSH, and CAT) following DOX-induced cardiac damage, aligning with the reported literature $[5,23,34,40]$.

DOX induces an inflammatory response in rat myocardium [41] and is believed to induce a series of inflammatory 


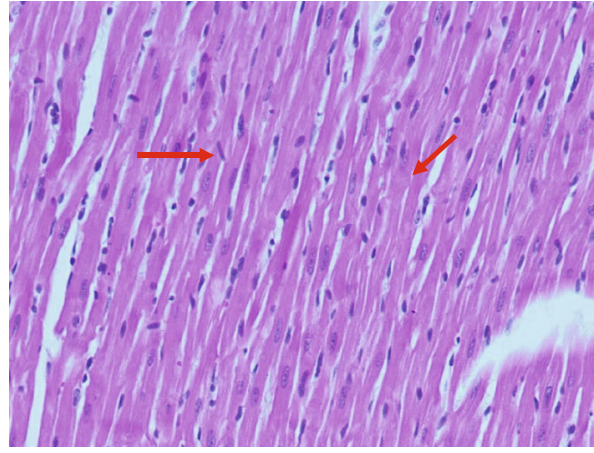

(a)

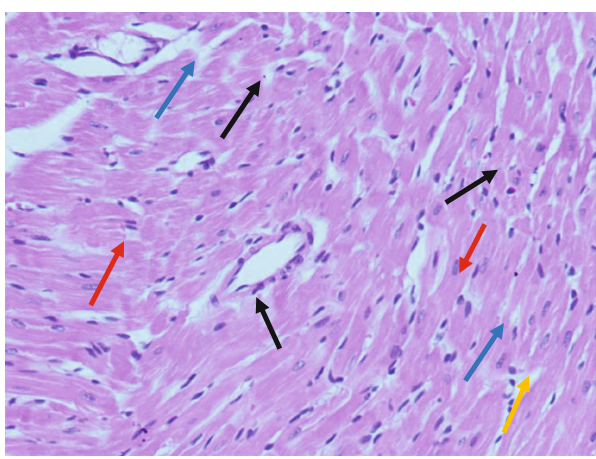

(c)

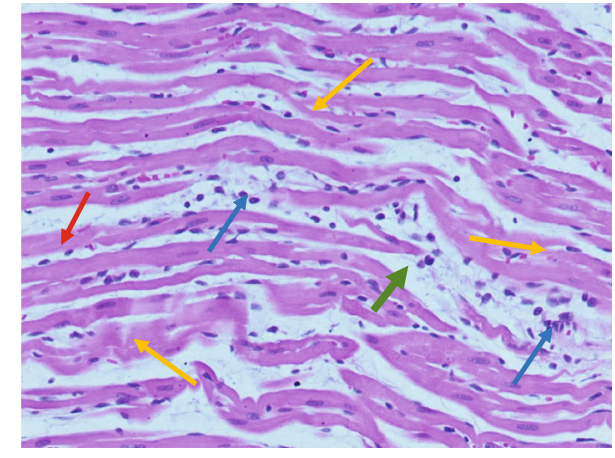

(b)

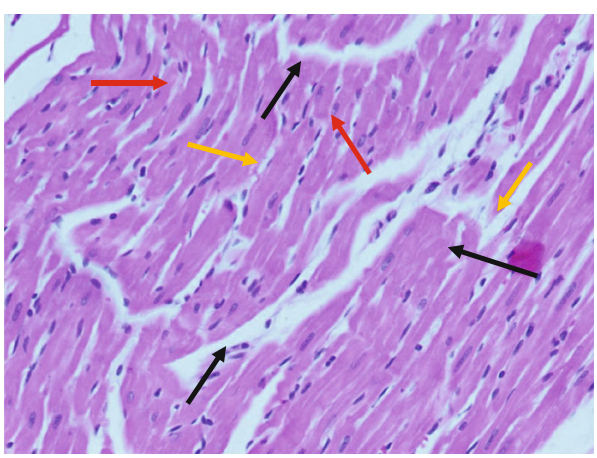

(d)

FIGURE 3: Effect of sinapic acid (SA) pretreatment on the histopathological changes in the cardiac tissue of DOX-treated rats. Photomicrographs of the myocardium tissue in (a) normal rats (group I) exhibiting normal cardio myofibril architecture (red arrow); (b) DOX-treated rats (group II) exhibiting perivascular cuffing (green arrow) of the vasa vasorum with intimal fibrosis, disrupted medial elastic fibers with diffuse interstitial fibrosis, myocytolysis (orange arrow), and myonecrosis (blue arrow); (c) DOX (15 mg/kg) $+\mathrm{SA}$ $(20 \mathrm{mg} / \mathrm{kg}$ )-treated rats (group III) showing decreased degree of myonecrosis (black arrow) and less infiltration of inflammatory cells; and (d) captopril (CAP; $30 \mathrm{mg} / \mathrm{kg})+\mathrm{SA}(20 \mathrm{mg} / \mathrm{kg})$-treated rats displaying reversal of myocardial damage based on the reduction in the degree of necrosis and minor infiltration of inflammatory cells. Heart tissues were stained with hematoxylin and eosin and visualized under a light microscope at 100x magnification.

reactions within the myocardium by upregulating NF- $\kappa \mathrm{B}$, inflammatory cytokines (TNF- $\alpha$ and IL- $\beta$ ), and MPO $[42,43]$. Consistent with previous reports, the findings of the current investigation support the primary role of inflammation in the pathogenesis of DOX-induced cardiotoxicity, validating the substantial increase in TNF- $\alpha$, IL$1 \beta$, and MPO levels in the DOX group compared to those in the normal control group. Although the primary pathway promoting the increases in inflammatory markers is yet to be fully elucidated, this might be caused by impaired antioxidant capacity, increased levels of free radicals, and the existence of lipid peroxidation, which together are initiating factors for the alterations. Certainly, it has been established that elevated levels of inflammatory markers are associated with enhanced oxidative stress, which is known to initiate inflammatory reactions by activating NF- $\kappa \mathrm{B}$, which regulates the release of cytokines [44].

DOX treatment generates ROS, disrupts endotheliumbased cardiac myocyte functions, and encourages the release of endothelial cell-derived ET-1, PGI2, NO, and NRG-1, causing cardiac toxicity [45]. Generation of NO leads to peroxynitrite ions that induce nitrostative stress in the myocardium, suppress myocardial contractility, and induce apo- ptosis $[46,47]$. DOX promotes the increase in NF- $\kappa \mathrm{B}$ p65, TNF- $\alpha$, and IL- $1 \beta$ levels in addition to increasing the ET-1 and NO levels. This reflects oxidative stress induced inflammatory responses. On the contrary, SA and CAP treatment decreased NF- $\kappa$ B expression and inhibited the downstream inflammatory cascade. Therefore, SA and CAP appear to possess potent anti-inflammatory effects. Our data corroborates with our previous studies, where SA was found to be an inhibitor of oxidative stress and inflammatory cytokines $[12,48]$. Moreover, NF- $\kappa \mathrm{B}$ p65 activation may lead to DOX-induced apoptosis in the myocardium [49]. In the current study, data from western blot analysis indicated that DOX may induce apoptotic and necrotic cellular injuries in the myocardium as indicated by the increase in Bax and caspase- 3 protein expression and decrease in the expression of the antiapoptotic protein, Bcl-2. SA and CAP administration significantly downregulated the expression of the proapoptotic proteins, Bax and caspase-3, and upregulated that of the antiapoptotic, Bcl-2. These findings reveal that the antiapoptotic activity, at least in part, is responsible for the cardioprotective effect of SA in DOX-induced cardiotoxicity in rats. These results corroborate with those of previous reports $[13,50,51]$. 


\section{Conclusions}

The present investigation demonstrates that SA administration can ameliorate DOX-induced cardiotoxicity by suppressing oxidative stress, inflammation, and apoptosis. The potential mechanism of its protective role is mediated by inhibition of inflammation and apoptosis via downregulation of NF- $\kappa$ B. Our findings above suggested that the use of SA could be expected to have synergistic efficacy and significant potential against cardiotoxicity induced by DOX. Thus, the approach of SA could be applied to treat or prevent DOXinduced cardiotoxicity in the future. SA may act as a cardioprotective agent that promotes the safe use of DOX in patients exposed to chemotherapy; further clinical studies were required to prove its clinical efficacy.

\section{Abbreviations}

$\begin{array}{ll}\text { SA: } & \text { Sinapic acid } \\ \text { DOX: } & \text { Doxorubicin } \\ \text { ROS: } & \text { Reactive oxygen species } \\ \text { CAP: } & \text { Captopril } \\ \text { LDH: } & \text { Lactate dehydrogenase } \\ \text { TNF- } \alpha: & \text { Tumor necrosis factor- } \alpha \\ \text { IL-1 } \beta \text { : } & \text { Interleukin-1 } \beta \\ \text { ET-1: } & \text { Endothelin-1 } \\ \text { NF- } \kappa \text { B: } & \text { Nuclear factor kappa-B } \\ \text { GPx: } & \text { Glutathione peroxidase } \\ \text { CAT: } & \text { Catalase } \\ \text { MPO: } & \text { Myeloperoxidase } \\ \text { ip: } & \text { Intraperitoneal } \\ \text { CK-MB: } & \text { Creatinine kinase MB fraction } \\ \text { NO: } & \text { Nitric oxide } \\ \text { MDA: } & \text { Malondialdehyde } \\ \text { SOD: } & \text { Superoxide dismutase. }\end{array}$

\section{Data Availability}

Data will be available from the corresponding author upon request.

\section{Conflicts of Interest}

The authors declare no conflict of interest.

\section{Authors' Contributions}

MAA. and YJ. conceptualized the study. MR., AA., and NH. helped in the methodology. AA. and MR. validated the study. AA. and MRK. did the formal analysis. AA., AA., and MR. did the investigation. MAA. and YJ. helped in resources. MRK. did the data curation. MAA and MR. wrote the original draft preparation. KMA., FAJ., AA., and MR. did the writing-review and editing. MAA. supervised the study. MAA. and YJ. helped in the project administration. MAA acquired funding.

\section{Acknowledgments}

The authors extend their appreciation to the Deanship of Scientific Research at King Saud University for funding this work through the Research Group Number, RG-1439-83.

\section{Supplementary Materials}

Graphical abstract (schematic representation of the present investigation). (Supplementary Materials)

\section{References}

[1] T. Šimůnek, M. Štěrba, O. Popelová, M. Adamcová, R. Hrdina, and V. Geršl, "Anthracycline-induced cardiotoxicity: overview of studies examining the roles of oxidative stress and free cellular iron," Pharmacological Reports, vol. 61, no. 1, pp. 154$171,2009$.

[2] F. Arcamone, G. Franceschi, S. Penco, and A. Selva, "Adriamycin (14-hydroxydaunomycin), a novel antitumor antibiotic," Tetrahedron Letters, vol. 10, no. 13, pp. 1007-1010, 1969.

[3] S. M. Swain, F. S. Whaley, and M. S. Ewer, "Congestive heart failure in patients treated with doxorubicin - a retrospective analysis of three trials," Cancer, vol. 97, no. 11, pp. 28692879, 2003.

[4] G. Minotti, P. Menna, E. Salvatorelli, G. Cairo, and L. Gianni, "Anthracyclines: molecular advances and pharmacologic developments in antitumor activity and cardiotoxicity," Pharmacological Reviews, vol. 56, no. 2, pp. 185-229, 2004.

[5] J. P. Zhang, L. Cui, X. Han et al., "Protective effects of tannic acid on acute doxorubicin-induced cardiotoxicity: involvement of suppression in oxidative stress, inflammation, and apoptosis," Biomedicine \& Pharmacotherapy, vol. 93, pp. 1253-1260, 2017.

[6] C. Myers, "The role of iron in doxorubicin-induced cardiomyopathy," Seminars in Oncology, vol. 25, no. 4, pp. 10-14, 1998.

[7] S. Y. Saad, T. A. Najjar, and A. C. Al-Rikabi, "The preventive role of deferoxamine against acute doxorubicin-induced cardiac, renal and hepatic toxicity in rats," Pharmacological Research, vol. 43, no. 3, pp. 211-218, 2001.

[8] J. Kluza, P. Marchetti, M. A. Gallego et al., "Mitochondrial proliferation during apoptosis induced by anticancer agents: effects of doxorubicin and mitoxantrone on cancer and cardiac cells," Oncogene, vol. 23, no. 42, pp. 7018-7030, 2004.

[9] S. Granados-Principal, J. L. Quiles, C. L. Ramirez-Tortosa, P. Sanchez-Rovira, and M. C. Ramirez-Tortosa, "New advances in molecular mechanisms and the prevention of adriamycin toxicity by antioxidant nutrients," Food and Chemical Toxicology, vol. 48, no. 6, pp. 1425-1438, 2010.

[10] D. S. Shin, K. W. Kim, H. Y. Chung, S. Yoon, and J. O. Moon, "Effect of sinapic acid against carbon tetrachloride-induced acute hepatic injury in rats," Archives of Pharmacal Research, vol. 36, no. 5, pp. 626-633, 2013.

[11] S. J. Roy and P. S. M. Prince, "Protective effects of sinapic acid on cardiac hypertrophy, dyslipidaemia and altered electrocardiogram in isoproterenol-induced myocardial infarcted rats," European Journal of Pharmacology, vol. 699, no. 1-3, pp. 213-218, 2013.

[12] M. A. Ansari, M. Raish, A. Ahmad et al., "Sinapic acid mitigates gentamicin-induced nephrotoxicity and associated oxidative/nitrosative stress, apoptosis, and inflammation in rats," Life Sciences, vol. 165, pp. 1-8, 2016. 
[13] T. Silambarasan, J. Manivannan, M. K. Priya, N. Suganya, S. Chatterjee, and B. Raja, "Sinapic acid protects heart against ischemia/reperfusion injury and H9c2 cardiomyoblast cells against oxidative stress," Biochemical and Biophysical Research Communications, vol. 456, no. 4, pp. 853-859, 2015.

[14] R. Nithya and S. Subramanian, "Antioxidant properties of sinapic acid: In vitro and in vivo approach," Asian Journal of Pharmaceutical and Clinical Research, vol. 10, no. 6, p. 255, 2017.

[15] G. Guillevin, M. D. Lardoux, and P. Corvol, "Effects of captopril on blood pressure, electrolytes, and certain hormones in hypertension," Clinical Pharmacology and Therapeutics, vol. 29, no. 6, pp. 699-704, 1981.

[16] M. Gheorghiade, L. De Luca, and R. O. Bonow, "Neurohormonal inhibition in heart failure: insights from recent clinical trials," American Journal of Cardiology, vol. 96, no. 12A, pp. 3L-9L, 2005.

[17] H. C. Yen, T. D. Oberley, S. Vichitbandha, Y. S. Ho, and D. K. S. Clair, "The protective role of manganese superoxide dismutase against adriamycin-induced acute cardiac toxicity in transgenic mice," Journal of Clinical Investigation, vol. 99, no. 5, pp. 1253-1260, 1996.

[18] O. H. Lowry, N. J. Rosebrough, A. L. Farr, and R. J. Randall, "Protein measurement with the Folin phenol reagent," Journal of Biological Chemistry, vol. 193, no. 1, pp. 265-275, 1951.

[19] R. A. Lawrence and R. F. Burk, "Glutathione peroxidase activity in selenium-deficient rat liver," Biochemical and Biophysical Research Communications, vol. 71, no. 4, pp. 952-958, 1976.

[20] C. Beauchamp and I. Fridovich, "Superoxide dismutase: improved assays and an assay applicable to acrylamide gels," Analytical Biochemistry, vol. 44, no. 1, pp. 276-287, 1971.

[21] R. F. Beers Jr. and I. W. Sizer, "A spectrophotometric method for measuring the breakdown of hydrogen peroxide by catalase," Journal of Biological Chemistry, vol. 195, no. 1, pp. 133-140, 1952.

[22] H. Towbin, T. Staehelin, and J. Gordon, "Electrophoretic transfer of proteins from polyacrylamide gels to nitrocellulose sheets: procedure and some applications," Proceedings of the National Academy of Sciences of the United States of America, vol. 76, no. 9, pp. 4350-4354, 1979.

[23] E. M. Mantawy, W. M. el-Bakly, A. Esmat, A. M. Badr, and E. el-Demerdash, "Chrysin alleviates acute doxorubicin cardiotoxicity in rats via suppression of oxidative stress, inflammation and apoptosis," European Journal of Pharmacology, vol. 728, pp. 107-118, 2014.

[24] G. Takemura and H. Fujiwara, "Doxorubicin-induced cardiomyopathy from the cardiotoxic mechanisms to management," Progress in Cardiovascular Diseases, vol. 49, no. 5, pp. 330-352, 2007.

[25] M. Yagmurca, E. Fadillioglu, H. Erdogan, M. Ucar, S. Sogut, and M. K. Irmak, "Erdosteine prevents doxorubicin-induced cardiotoxicity in rats," Pharmacological Research, vol. 48, no. 4, pp. 377-382, 2003.

[26] C. F. Thorn, C. Oshiro, S. Marsh et al., "Doxorubicin pathways: pharmacodynamics and adverse effects," Pharmacogenetics and Genomics, vol. 21, no. 7, pp. 440-446, 2011.

[27] L. Gille and H. Nohl, "Analyses of the molecular mechanism of adriamycin-induced cardiotoxicity," Free Radical Biology and Medicine, vol. 23, no. 5, pp. 775-782, 1997.

[28] M. F. Andreasen, A. K. Landbo, L. P. Christensen, A. Hansen, and A. S. Meyer, "Antioxidant effects of phenolic rye (Secale cereale L.) extracts, monomeric hydroxycinnamates, and ferulic acid dehydrodimers on human low-density lipoproteins," Journal of Agricultural and Food Chemistry, vol. 49, no. 8, pp. 4090-4096, 2001.

[29] N. Niciforovic and H. Abramovic, "Sinapic acid and its derivatives: natural sources and bioactivity," Comprehensive Reviews in Food Science and Food Safety, vol. 13, no. 1, pp. 34-51, 2014.

[30] T. Silambarasan, J. Manivannan, B. Raja, and S. Chatterjee, "Prevention of cardiac dysfunction, kidney fibrosis and lipid metabolic alterations in l-NAME hypertensive rats by sinapic acid-role of HMG-CoA reductase," European Journal of Pharmacology, vol. 777, pp. 113-123, 2016.

[31] I. Sharma, M. Aaradhya, M. Kodikonda, and P. R. Naik, “Antihyperglycemic, antihyperlipidemic and antioxidant activity of phenolic rich extract of Brassica oleraceae var gongylodes on streptozotocin induced Wistar rats," Springerplus, vol. 4, article 212, 2015.

[32] H. Gandhi, V. B. Patel, N. Mistry, N. Patni, J. Nandania, and R. Balaraman, "Doxorubicin mediated cardiotoxicity in rats: protective role of felodipine on cardiac indices," Environmental Toxicology and Pharmacology, vol. 36, no. 3, pp. 787-795, 2013.

[33] A. Ascensão, J. Magalhães, J. Soares et al., "Endurance training attenuates doxorubicin-induced cardiac oxidative damage in mice," International Journal of Cardiology, vol. 100, no. 3, pp. 451-460, 2005.

[34] A. V. Swamy, S. Gulliaya, A. Thippeswamy, B. C. Koti, and D. V. Manjula, "Cardioprotective effect of curcumin against doxorubicin-induced myocardial toxicity in albino rats," Indian Journal of Pharmacology, vol. 44, no. 1, pp. 73-77, 2012.

[35] K. Ytrehus, Y. Liu, A. Tsuchida et al., "Rat and rabbit heart infarction - effects of anesthesia, perfusate, risk zone, and method of infarct sizing," American Journal of PhysiologyHeart and Circulatory Physiology, vol. 267, no. 6, pp. H2383H2390, 1994.

[36] I. E. Takś, B. Matkovics, S. I. Varga, P. Homolay, G. Fehér, and T. Seres, "Study of the myocardial antioxidant defence in various species," Pharmacological Research, vol. 25, pp. 177-178, 1992.

[37] M. A. Ibrahim, O. M. Ashour, Y. F. Ibrahim, H. I. el-Bitar, W. Gomaa, and S. R. Abdel-Rahim, "Angiotensin-converting enzyme inhibition and angiotensin $\mathrm{AT}_{1}$-receptor antagonism equally improve doxorubicin-induced cardiotoxicity and nephrotoxicity," Pharmacological Research, vol. 60, no. 5, pp. 373-381, 2009.

[38] E. Balli, U. O. Mete, A. Tuli, O. Tap, and M. Kaya, "Effect of melatonin on the cardiotoxicity of doxorubicin," Histology and Histopathology, vol. 19, no. 4, pp. 1101-1108, 2004.

[39] T. Silambarasan, J. Manivannan, M. Krishna Priya, N. Suganya, S. Chatterjee, and B. Raja, "Sinapic acid prevents hypertension and cardiovascular remodeling in pharmacological model of nitric oxide inhibited rats," PLoS One, vol. 9, no. 12, article e115682, 2014.

[40] J. Das, J. Ghosh, P. Manna, and P. C. Sil, “Taurine suppresses doxorubicin-triggered oxidative stress and cardiac apoptosis in rat via up-regulation of PI3-K/Akt and inhibition of p53, p38-JNK," Biochemical Pharmacology, vol. 81, no. 7, pp. 891-909, 2011.

[41] J. Zhu, J. Zhang, L. Zhang et al., "Interleukin-1 signaling mediates acute doxorubicin-induced cardiotoxicity," Biomedicine \& Pharmacotherapy, vol. 65, no. 7, pp. 481-485, 2011. 
[42] T. A. Abd El-Aziz, R. H. Mohamed, H. F. Pasha, and H. R. Abdel-Aziz, "Catechin protects against oxidative stress and inflammatory-mediated cardiotoxicity in adriamycin-treated rats," Clinical and Experimental Medicine, vol. 12, no. 4, pp. 233-240, 2012.

[43] A. A. Hamza, M. M. Ahmed, H. M. Elwey, and A. Amin, "Melissa officinalis protects against doxorubicin-induced cardiotoxicity in rats and potentiates its anticancer activity on MCF-7 cells," PLoS One, vol. 11, no. 11, article e0167049, 2016.

[44] Z. Q. Wang, M. T. Chen, R. Zhang, Y. Zhang, W. Li, and Y. G. $\mathrm{Li}$, "Docosahexaenoic acid attenuates doxorubicin-induced cytotoxicity and inflammation by suppressing NF- $\kappa \mathrm{B} / \mathrm{i}$ NOS/NO signaling pathway activation in H9C2 cardiac cells," Journal of Cardiovascular Pharmacology, vol. 67, no. 4, pp. 283-289, 2016.

[45] A. Z. Luu, B. Chowdhury, M. al-Omran, H. Teoh, D. A. Hess, and S. Verma, "Role of endothelium in doxorubicin-induced cardiomyopathy," JACC: Basic to Translational Science, vol. 3, no. 6, pp. 861-870, 2018.

[46] I. N. Mungrue, R. Gros, X. You et al., "Cardiomyocyte overexpression of iNOS in mice results in peroxynitrite generation, heart block, and sudden death," The Journal of Clinical Investigation, vol. 109, no. 6, pp. 735-743, 2002.

[47] H. Doods and D. Wu, "Sabiporide reduces ischemia-induced arrhythmias and myocardial infarction and attenuates ERK phosphorylation and iNOS induction in rats," BioMed Research International, vol. 2013, Article ID 504320, 9 pages, 2013.

[48] M. Raish, A. Ahmad, M. Ahmad Ansari et al., "Sinapic acid ameliorates bleomycin-induced lung fibrosis in rats," Biomedicine \& Pharmacotherapy, vol. 108, pp. 224-231, 2018.

[49] D. Li, J. Li, Y. An, Y. Yang, and S. Q. Zhang, "Doxorubicininduced apoptosis in $\mathrm{H} 9 \mathrm{c} 2$ cardiomyocytes by NF- $\kappa \mathrm{B}$ dependent PUMA upregulation," European Review for Medical and Pharmacological Sciences, vol. 17, no. 17, pp. 2323-2329, 2013.

[50] B. D. Sahu, J. M. Kumar, M. Kuncha, R. M. Borkar, R. Srinivas, and R. Sistla, "Baicalein alleviates doxorubicin-induced cardiotoxicity via suppression of myocardial oxidative stress and apoptosis in mice," Life Sciences, vol. 144, pp. 8-18, 2016.

[51] S. Shabalala, C. J. F. Muller, J. Louw, and R. Johnson, "Polyphenols, autophagy and doxorubicin-induced cardiotoxicity," Life Sciences, vol. 180, pp. 160-170, 2017. 